\section{Cancellation Policy}

Written notice of cancellation received by June 2,1989 , will be honored subject to a $\$ 15$ cancellation charge. No refunds for cancellation after June 2 . The Preconference may be canceled if there is insufficient registration. If it is canceled, ACRL cannot be responsible for cancellation or change charges assessed by airlines or travel agencies.

None of the registration fee is for meal or entertainment expenses. You should consult your tax adviser regarding the deductibility for federal income tax purposes of various components of the registration fee.

\title{
ACRL meetings in Dallas
}

\section{A tentative schedule for ALA's Dallas Conference, June 24-29, 1989.}

\section{ACRL BOARD OF DIRECTORS}

Orientation breakfast: Saturday, June 24, 8:00-9:00 a.m.

First meeting: Saturday, June 24, 2:00-4:00 p.m.

Second meeting: Tuesday, June 27, 2:00-5:30 p.m.

\section{ACRL GENERAL}

Orientation for new chairs: Friday, June 23, 4:30-5:30 p.m.

President's Program/Membership: Monday, June 26, 2:00-5:00 p.m.; reception, 5:00-6:00 p.m.

\section{ACRL DIVISIONAL C OMMITTEES}

Academic Library Statistics: Saturday, June 24, 8:00-9:00 a.m.; Monday, June 26, 8:00-9:00 a.m.

Academic Status: Sunday, June 25, 9:00-11:00 a.m.; Monday, June 26, 9:00-11:00 a.m.; Tuesday, June 27, 9:00-11:00 a.m.

Appointments and Nominations: Saturday, June 24, 9:00-11:00 a.m.; Monday, June 26, 2:00-6:00 p.m.*

Audiovisual: Sunday, June 25, 9:00-11:00 a.m.; Monday, June 26, 9:00-11:00 a.m.

ACRL/AECT Joint Committee on Two-Year Colleges: Friday, June 23, 2:00-5:30 p.m.; Monday, June 26, 4:00-5:30 p.m.
Budget and Finance: Saturday, June 24, 9:30 a.m.-12:30 p.m.; Sunday, June 25, 9:30 a.m.-12:30 p.m., 2:00-5:30 p.m.; Tuesday, June 27, 8:00 a.m.-12:30 p.m.

Constitution and Bylaws: Saturday, June 24, 8:00-11:00 a.m.; Monday, June 26, 8:00-11:00 a.m.

Copyright: Saturday, June 24, 8:30-11:00 a.m.; Sunday, June 25, 9:30-11:00 a.m.

Extended Campus Library Services Guidelines: Sunday, June 25, 8:00-10:00 p.m.; Tuesday, June 27, 9:00-11:00 a.m.

Faculty Advisory Committee Orientation Material Task Force: Sunday, June 25, 9:00-11:00 a.m.

Financial Development Task Force: Monday, June 26, 9:30 a.m.-12:00 noon.

International Relations Task Force: Saturday, June 24, 9:00-11:00 a.m.; Sunday, June 25, 9:00-11:00 a.m.

Library Access Task Force: Sunday, June 25, 8:00-10:00 p.m.

\section{Correction}

The Hugh Atkinson Memorial Award will be presented to Thomas Michalak at a reception in his honor on Sunday, June 25, 4:30-6:00 p.m., during the ALA Annual Conference in Dallas. 
Library School Curriculum Task Force: Monday, June 26, 8:30 a.m. $-12: 30$ p.m.

Membership: Monday, June 26, 9:30 a.m.-12:30 p.m.

Minority Recruitment Task Force: Monday, June 26, 9:30-11:00 a.m.

Non-Librarian Professionals in Academic Libraries Task Force: Friday, June 23, 3:00-5:00 p.m.; Monday, June 26, 9:00-11:00 a.m.

Paraprofessionals in Academic Libraries Task Force: Sunday, June 25, 8:00 a.m.-12:00 noon.

Performance Measures for Academic Libraries: Saturday, June 24, 9:30 a.m.-12:30 p.m.; Monday, June 26, 9:30 a.m.-12:30 p.m.

Planning: Saturday, June 24, 2:00-4:00 p.m.; Monday, June 26, 9:00 a.m. $-12: 30$ p.m.

Professional Association Liaison: Sunday, June 25, 2:00-4:00 p.m.; Monday, June 26, 9:00-11:00 a.m. (second meeting is a closed meeting).

Professional Education: Sunday, June 25, 2:00-4:00 p.m.; Monday, June 26, 9:30-11:00 a.m.

Professional Ethics Task Force: Sunday, June 25, 9:30-11:00 a.m.

Publications: Saturday, June 24, 9:00-11:00 a.m.; Sunday, June 25, 2:00-4:00 p.m.

Research: Saturday, June 24, 9:00-11:00 a.m.; Monday, June 26, 8:30-11:30 a.m.

Sources of Revenue in Academic Libraries Task Force: Sunday, June 25, 2:00-4:00 p.m.; Tuesday, June 27, 9:30-11:00 a.m.

Standards and Accreditation: Saturday, June 24, 9:00-11:00 a.m.; Monday, June 26, 9:00-11:00 a.m.

\section{ACRL CHAPTERS COUNCIL}

Business and Orientation: Sunday, June 25, 8:30 a.m.-12:30 p.m.

\section{ACRL DISCUSSION GROUPS}

Australian Studies: Sunday, June 25, 11:30 a.m. $-12: 30$ p.m.

Black Studies Librarianship: Saturday, June 24, 2:00-4:00 p.m.

Electronic Library Development in Academic Libraries: Saturday, June 24, 11:30 a.m.-12:30 p.m.

English and American Literature-Program: Sunday, June 25, 2:00-5:30 p.m.

Extended Campus Library Services: Monday, June 26, 9:30-11:00 a.m.

Fee-Based Information Service Centers in Academic Libraries: Monday, June 26, 9:00-11:00 a.m.; Tuesday, June 27, 9:00-11:00 a.m.

Heads of Reader/Public Services: Sunday, June 25, 4:30-6:00 p.m.

Home Economics/Human Ecology Librarians: Sunday, June 25, 4:30-5:30 p.m.
Journal Costs in Academic Libraries: Sunday, June 25, 9:30-11:00 a.m.

Librarians of Library Science Collections: Saturday, June 24, 9:00-11:00 a.m.

Microcomputer Services in Academic Libraries: Sunday, June 25, 2:00-4:00 p.m.

Personnel Administrators and Staff Development Officers: Saturday, June 24, 9:30-11:00 a.m.; Sunday, June 25, 9:30-11:30 a.m.; Monday, June 26, 9:30-11:00 a.m.

Popular Culture and Libraries: Monday, June 26, 9:00-11:00 a.m.

Public Relations in Academic Libraries: Sunday, June 25, 2:00-5:30 p.m.

Research: Sunday, June 25, 2:00-4:00 p.m.

Undergraduate Librarians: Monday, June 26, 9:30 a.m. $-12: 30$ p.m.

\section{ACRL EDITORIAL BOARDS}

Choice: Saturday, June 24, 2:00-4:00 p.m.

College \& Research Libraries: Sunday, June 25, 9:00-11:00 a.m.

College \& Research Libraries News: Sunday, June 25, 8:30-9:30 a.m.

Publications in Librarianship: Monday, June 26, 8:30-11:00 a.m.

Rare Books \& Manuscripts Librarianship: Saturday, June 24, 2:00-4:00 p.m.

\section{ACRL SECTIONS}

Activity Sections Council

Meeting: Tuesday, June 27, 11:30 a.m.-12:30 p.m.

\section{Anthropology and Sociology Section}

Program: Sunday, June 26, 9:30 a.m.-12:30 p.m.

Executive: Saturday, June 24, 2:00-4:00 p.m.; Monday, June 26, 2:00-5:30 p.m.

Bibliography: Saturday, June 24, 9:00-11:00 a.m.; Monday, June 26, 9:00-11:00 a.m.

Conference Program Planning-Atlanta, 1991: Saturday, June 24, 8:00-9:00 a.m.; Monday, June 26, 8:00-9:00 a.m.

Conference Program Planning-Chicago, 1990: Saturday, June 24, 8:00-9:00 a.m.; Monday, June 26, 8:00-9:00 a.m.

Nominating: Monday, June 26, 11:30 a.m.-12:30 p.m.*

Publications: Saturday, June 24, 11:30 a.m.-12:30 p.m.; Sunday, June 25, 2:00-4:00 p.m.

Review and Planning: Sunday, June 25, 4:30-5:30 p.m.

\section{Art Section}

Program: Sunday, June 25, 2:00-5:00 p.m. Executive: Sunday, June 25, 9:30-11:00 a.m. Committees: Saturday, June 24, 2:00-4:00 p.m. Membership: Monday, June 26, 2:00-4:00 p.m.

Technology in the Arts: Monday, June 26, 9:30 a.m. $-12: 30$ p.m. 


\section{Asian and African Section}

Program: Monday, June 26, 9:30 a.m.-12:30 p.m. Executive: Sunday, June 25, 9:30-11:30 a.m.

\section{Bibliographic Instruction Section}

Program: Sunday, June 25, 2:00-5:30 p.m.

Executive: Saturday, June 24, 8:00-9:00 a.m.; Tuesday, June 27, 9:30 a.m.-12:30 p.m.

Advisory Council: Saturday, June 24, 9:30-11:00 a.m.; Monday, June 26, 2:00-4:00 p.m.

Communication: Saturday, June 24, 2:00-4:00 p.m.

Computer Concerns: Sunday, June 25, 9:30 a.m.-12:30 p.m.

Conference Program Planning-Dallas, 1989: Saturday, June 24, 2:00-4:00 p.m.

Conference Program Planning-Chicago, 1990: Sunday, June 25, 9:00-11:00 a.m.

Continuing Education: Saturday, June 24, 2:00-4:00 p.m.

Education for Bibliographic Instruction: Sunday, June 25, 9:30 a.m.-12:30 p.m.

Miriam Dudley BI Librarian of the Year Award: Monday, June 26, 11:30 a.m.-12:30 p.m.*

Nominating: Monday, June 26, 11:30 a.m.-12:30 p.m.*

Planning: Sunday, June 25, 9:00-11:00 a.m.

Policy: Saturday, June 24, 2:00-4:00 p.m.

Think Tank: Wednesday, June 21, 8:00-10:00 p.m.; Thursday, June 22, 8:00 a.m.-12:30 p.m., 2:00-5:30 p.m., 8:00-10:00 p.m.; Friday, June 23, 9:30 a.m. $-12: 30$ p.m., 2:00-4:00 p.m.

\section{College Libraries Section}

Program: Sunday, June 25, 9:30 a.m.-12:30 p.m.

Executive: Saturday, June 24, 9:00 a.m.-12:30 p.m.; Tuesday, June 27, 11:30 a.m.-1:00 p.m.

CLIP Notes: Monday, June 26, 2:00-4:00 p.m.

Historical Commission: Saturday, June 24, 2:00-4:00 p.m.; Monday, June 26, 8:00 a.m. $-12: 30$ p.m.

Library Directors Discussion Group: Sunday, June 25, 2:00-4:00 p.m.

Membership: Sunday, June 25, 9:00-9:30 a.m.

Member Reception: Sunday, June 25, 4:00-6:00 p.m.

National Advisory Council: Sunday, June 25, 8:30-9:00 a.m.

Newsletter: Saturday, June 24, 2:00-4:00 p.m.

Standards: Saturday, June 24, 2:00-4:00 p.m.; Tuesday, June 26, 9:00-11:00 a.m.

Community and Junior College Libraries Section

Program: Saturday, June 24. 9:30 a.m.-12:30 p.m.

Committees: Sunday, June 25, 8:30 a.m.-12:30 p.m.

\section{Education and Behavioral Sciences Section}

Program: Monday, June 26, 9:30 a.m.-12:30 p.m. Executive: Tuesday, June 27, 8:00 a.m.-12:30 p.m.

BI for Educators: Sunday, June 25, 9:00 a.m. $-12: 30$ p.m.

Conference Program Planning-Dallas, 1989: Sat-

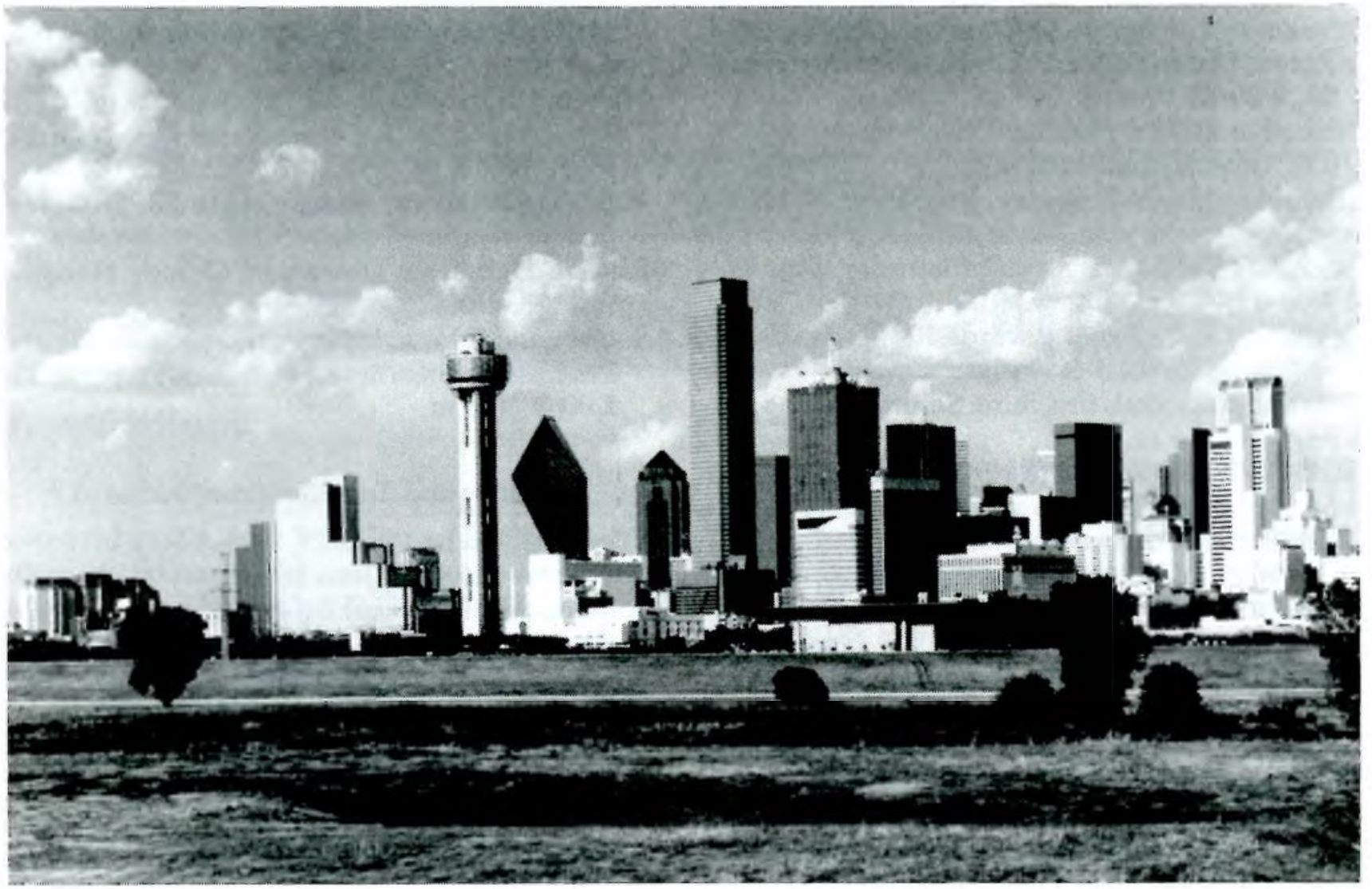

Beautiful downtown Dallas. 
urday, June 24, 2:00-4:00 p.m.

Conference Program Planning-Chicago, 1990:

Saturday, June 24, 9:00-11:00 a.m.

Curriculum Materials: Sunday, June 25, 2:00-5:30 p.m.

Psychology/Psychiatry: Sunday, June 25, 8:00-11:00 a.m.; Monday, June 26, 2:00-5:00 p.m.

Publications: Monday, June 26, 8:00-9:00 a.m.

\section{Law and Political Science Section}

Executive: Saturday, June 24, 8:00-9:00 a.m.; Tuesday, June 27, 8:00-9:00 a.m.

Bibliographies: Monday, June 26, 9:30-11:00 a.m. Business: Tuesday, June 27, 9:00-11:00 a.m.

Conference Program Planning-Chicago, 1990: Saturday, June 24, 9:30-11:00 a.m.

Nominating: Sunday, June 25, 4:30-5:30 p.m.*

Publications: Saturday, June 24, 9:30-11:00 a.m.

Review and Planning: Sunday, June 25, 9:30-11:00 a.m.

\section{Rare Books and Manuscripts Section}

Program: Sunday, June 25, 2:00-5:30 p.m.

Executive: Sunday, June 25, 11:30 a.m.-12:30 p.m.; Monday, June 26, 9:30 a.m. $-12: 30$ p.m.

Cambridge Conference Planning: Sunday, June 25, 9:00-11:00 a.m.

Conference Program Planning-Chicago, 1990: Saturday, June 24, 11:30 a.m.-12:30 p.m.

Conservators' Collations: Saturday, June 24, 2:00-4:00 p.m.

Curators and Conservators Discussion Group: Saturday, June 24, 11:30 a.m.-12:30 p.m.

Developing Guidelines for Borrowing Special Collection Materials for Exhibition: Sunday, June 25, 9:00-11:00 a.m.

Education and Professional Development: Saturday, June 24, 2:00-4:00 p.m.

Ethical Standards: Saturday, June 24, 9:00-11:00 a.m.

Exhibition Catalogues Awards: Saturday, June 24, 9:00-11:00 a.m.*

Exhibition Catalogues Awards Reception: Sunday, June 25, 5:30-6:30 p.m.

Gifts and Appraisals Brochure: Saturday, June 24, 9:00-11:00 a.m.

GODORT/MAGERT/RBMS Joint Committee: Sunday, June 25, 11:30 a.m.-12:30 p.m.

Guidelines on Archives and Manuscripts Review: Saturday, June 24, 2:00-4:00 p.m.

Information Exchange: Sunday, June 25, 8:00-10:00 p.m.

Legislative Information: Saturday, June 24, 2:00-4:00 p.m.

Literary Rights: Sunday, June 25, 9:00-11:00 a.m. Manuscripts and Other Formats: Saturday, June 24, 9:00-11:00 a.m.

MARC for Special Collections Discussion Group: Saturday, June 24, 11:30 a.m.-12:30 p.m.

Nominating: Saturday, June 24, 8:00-10:00 p.m. *
Orientation Manual: Saturday, June 24, 11:30 a.m. $-12: 30$ p.m.

Preconference Program Planning-Chicago, 1990: Saturday, June 24, 8:00-9:00 a.m.

Publications: Sunday, June 25, 9:00-11:00 a.m.

Records Retention: Saturday, June 24, 9:00-11:00 a.m.

Security: Saturday, June 24, 11:30 a.m.-12:30 p.m.; Sunday, June 25, 9:00-11:00 a.m.

Standards: Saturday, June 24, 9:00-11:00 a.m., 2:00-4:00 p.m., 8:00-10:00 p.m.; Sunday, June 25, 9:00-11:00 a.m.

Statistical Survey Feasibility: Saturday, June 24, 2:00-4:00 p.m.

\section{Science and Technology Section}

Program: Tuesday, June 27, 9:30 a.m.-12:30 p.m. Comparison of Science and Engineering Libraries: Saturday, June 24, 9:30 a.m.-12:30 p.m.

Conference Proceedings Task Force: Saturday, June 24, 9:00-11:00 a.m.

Conference Program Planning-Dallas, 1989: Sunday, June 25, 8:30 a.m.-12:30 p.m.

Conference Program Planning-Chicago, 1990: Monday, June 26, 8:30 a.m.-12:30 p.m.

Council: Friday, June 23, 8:00-10:00 p.m.; Monday, June 26, 11:30 a.m.-12:30 p.m.

Discussion Group: Saturday, June 24, 2:00-4:00 p.m.

Forum on Emerging Issues and Research Task Force: Monday, June 26, 9:00-11:00 a.m.

Legislation: Sunday, June 25, 9:00-11:00 a.m.

Membership: Sunday, June 25, 9:00-11:00 a.m.

Newsletter: Sunday, June 25, 8:00-10:00 p.m.

Nominating: Sunday, June 25, 9:00-11:00 a.m. *

Oberly Award: Sunday, June 25, 9:00-11:00 a.m.*

Planning: Sunday, June 26, 2:00-4:00 p.m.

Preconference Task Force: Saturday, June 24, 9:00-11:00 a.m.

Publisher Relations: Sunday, June 25, 2:00-4:00 p.m.

Science Databases Discussion Group: Monday, June 26, 2:00-4:00 p.m.

Subject and Bibliographic Access to Scientific and Technical Information: Sunday, June 25, 2:00-4:00 p.m.

\section{Slavic and East European Section}

Program: Sunday, June 25, 2:00-4:00 p.m.

Executive: Monday, June 26, 2:00-4:00 p.m.

Automated Bibliographic Control: Monday, June 26, 9:00-11:00 a.m.

Conference Program Planning-Chicago, 1990: Sunday, June 25, 11:30 a.m.-12:30 p.m.

Continuing Education on Slavic and East European Librarianship in North America: Sunday, June 25, 9:30-11:00 a.m.

Membership: Sunday, June 25, 4:30-5:30 p.m.

Newsletter: Monday, June 26, 11:30 a.m.-12:30 p.m. 
Nominating: Sunday, June 25, 8:00-9:00 a.m. ${ }^{*}$

University Libraries Section

Program: Saturday, June 24, 2:00-4:00 p.m.

Steering: Saturday, June 24, 9:00-11:00 a.m.; Monday, June 26, 9:00-11:00 a.m.

Conference Program Planning-Chicago, 1990: Saturday, June 24, 11:30 a.m.-12:30 p.m.; Tuesday, June 27, 8:00-9:00 a.m.

Conference Program Planning-Dallas, 1989: Saturday, June 24, 9:30-11:00 a.m.

Current Topics Planning: Sunday, June 25, 9:00-11:00 a.m., 2:00-4:00 p.m.

Guidelines for Branch Libraries Review: Saturday, June 24, 2:00-4:00 p.m.; Tuesday, June 27, 2:00-4:00 p.m.

Nominating: Sunday, June 25, 11:30 a.m.-12:30 p.m.*

Organization and Bylaws: Sunday, June 25, 9:30-11:00 a.m.; Tuesday, June 27, 9:30-11:00 a.m.

Performance Measures: Sunday, June 25, 9:30-11:00 a.m.; Tuesday, June 27, 9:30-11:00 a.m.

Policy and Planning: Sunday, June 25, 2:00-4:00 p.m.; Tuesday, June 27, 8:30-11:00 a.m.

Promotion and Publications: Sunday, June 25, 9:00-11:00 a.m.; Tuesday, June 27, 9:00-11:00 a.m.

\section{Western European Specialists Section}

Program: Monday, June 26, 9:30 a.m.-12:30 p.m. Executive: Saturday, June 24, 2:00-4:00 p.m.; Tuesday, June 27, 2:00-4:00 p.m.

Classical, Medieval and Renaissance Discussion Group: Sunday, June 25, 11:30 a.m.-12:30 p.m. College and Medium-Sized Libraries Discussion
Group: Sunday, June 25, 9:30-11:00 a.m.

Conference Program Planning-Chicago, 1990:

Tuesday, June 27, 8:00-9:00 a.m.

Continuing Education: Sunday, June 25, 9:30-11:00 a.m.

Florence Conference Proceedings: Tuesday, June 27, 11:30 a.m.-12:30 p.m.

General Discussion Group: Monday, June 26, 2:00-4:00 p.m.

Germanists Discussion Group: Sunday, June 25, 9:30-11:00 a.m.

Martinus Nijhoff International West European Specialist Study Grant Award: Sunday, June 25, 4:30-5:30 p.m.*

Nominating: Monday, June 26, 8:00-9:00 p.m. ${ }^{*}$

Publications: Sunday, June 25, 8:00-10:00 p.m.

Publications-Directory: Sunday, June 25, 8:00-9:00 a.m.

Publications-Newsletter: Sunday, June 25, 4:30-5:30 p.m.

Research and Planning: Tuesday, June 27, 9:00-11:00 a.m.

\section{Women's Studies Section}

Program: Sunday, June 25, 9:30 a.m.-12:30 p.m.

Executive: Friday, June 23, 2:00-4:00 p.m.

Executive-Section Review: Tuesday, June 27, 2:00-5:30 p.m.

Communications: Sunday, June 25, 4:00-5:30 p.m.

Conference Program Planning-Chicago, 1990: Saturday, June 24, 9:30-11:00 a.m.

General Membership Meeting: Monday, June 26, 9:30-11:00 a.m., 2:00-4:00 p.m.

Nominating: Sunday, June 25, 4:00-5:30 p.m.*

Technical Services: Sunday, June 25, 4:00-5:30 p.m.

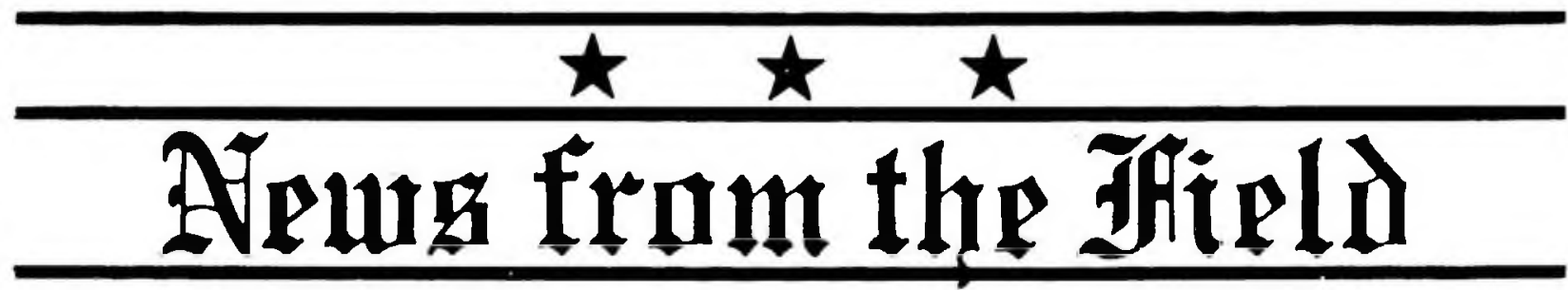

\section{Acquisitions}

- Texas A\&M University, College Station, has acquired more than 700 Chinese-language volumes of literature, history, political science, art, travel, and science as a gift from the state education commission of China in honor of the university's commitment to attracting and educating Chinese students. This is the first extensive gift of foreign-language materials presented to the university's Sterling C. Evans Library.

-The University of British Columbia, Vancouver, has acquired the personal papers of B.C. author Jane Rule. The papers range from 1947 to 1984 and include notes, manuscripts, and corre- spondence relating to her published and unpublished novels and short stories; biographical and autobiographical material, college papers, teaching records, non-fiction manuscripts; and personal and professional correspondence. Rule has published many books including Desert of the Heart (1964), Contract with the World (1980), and Memory Board (1987).

- The University of California, Berkeley, has acquired the surviving papers of one of France's top scientists at the time of the French Revolution, Pierre Simon Laplace (1749-1827). The 25 boxes of scientific and personal papers are the literary remains of a devastating fire in 1925 at the Laplace 